air about them, and produce upward currents or a buoying of the athermanous particles by dilation of the air in contact with them.

This affords a complete explanation of cloud-flotation and the flotation of fine dust-particles. For some years I have been in the habit of watching clouds, and by the use of the above theory have very often been able to account for forms, dimensions, and movements which I could not otherwise explain. Some fours ago I explained the above ideas to the Chief Meteorological Officer of the United States Signal Service, and received from him suggestions which have since afforded me the means of much pleasure in observing the locations and forms and movements of clouds, and although these irregular masses are subject to many $\mathrm{com}-$ plicating circumstances, I have never yet observed anything tending to weaken this theory of fotation, but have made many hundreds of olsecrvations tencling to confirm it.

I trust that it will be taken for granted that I do not wish to attack the hypothesis of Dr. Lodge and Mr. Clark, that heated bodies "bombard" and drive away approaching particles. My object is simply to show that, as it seems to me, the theory of particles buoyed up by a locally heated fluid, when considered in connection with well-known principles of radiation, \&c., is sufficient to account for the phenomenon of the "dust-free coat" rlescribed in the article alluded to.

Referring to the figures on p. 6I2, an ascending current is shown in the neighbourhood of the pipe or $\operatorname{rod}$ in Fig. I. The theory which I have sketched would indicate that this current had been set up in great measure by the indirect action of the heated tube or rod upon the surrounding air.

I should contend that the dust-free coat may be explained as follows :-

A given particle which may be assumed to be directly below the rod is heated by radiation from the rod. It in turn heats and expands the air in contact with it; the particle with a coat of adherent air becomes lighter than the surrounding atmosphere, and the mote, with its jacket of expanded air, ascends towards the rod. As it reaches the point marked "slow moving" in the figure, it begins to find itself in air which has been heated directly by contact with the rod, and distributed near it by the small "circular" currents which always surround a blunt obstacle in a stream of fluid. At the outer limit of the "dust-free coat" the particle or mote is arrested because it has come to a point where the air is so warm that the mote can no longer heat its jacket enough hotter than its surroundings to cause buoyancy. It is arrested because it has reached a point where the surroxnding medium is as light as its own air-float, much as cork is arrested at a surface of water.

The mote with its warm air jacket could ascend through cool and therefore heavy air, but the air warmed by contact with the pipe is too light to float it.

The dark "tail" above the rod, or tube, is the upstreaming dust-fiee air, warmed by the tube, and too light to carry motes, or in which motes have not been carried by any current.

The report of the lecture contains within itself some very striking confirmations of this theory. For example, Dr. Lodge tells us that at a high temperature the dust-free coat is thicker than at low ones. This is according to the theory of flotation as above set forth, because an approaching mote would sooner meet the increased body of air warmed by contact with the tube to a point sufficient to destroy the buoyancy of the mote and its jacket. Again, hydrogen is a light gas having a very high specific heat; hence according to this theory the mote would need more heat and more difference of temperature to float than in air, and consequently should not be able to float up to as near the rod. Now, Dr. Lodge states that "in hydrogen it [the dust-free coat] is thicker than in air." With a surrounding meclium of carbonic acid, less heat and less flotation are required for the mote, as the gas is heavier and of lower specific heat, and, quite in accord with the theory, the dust-free coat "is thinner" than in air or hydrogen. Again, Dr. Lodge states that the dust-free coat is set up by a "difference of a degree or two," and it would apparently require a much more complicated theory than the simple one here advanced to account for this on the bombardment hypothesis, as the action has been shown to be-

(I) Affected by the medium as to thickness of coat

(2) Obtainable at different temperatures in the rod

(3) Apparently dependent, not on the actual temperature of the rod, but on the differences in temperature between the rod and its surrounding dust-containing fluid.
The bchaviour of cool rods or plates, as stated, is also in accord with this theory. A mote coming within the influence of the plate or rod is cooled by radiation and loses buoyancy in its air jacket. If above the plate, it therefore falls upon it ; if below, it drops away. Dr. Lodge does not explain how a cool plate "bombards" the motes and drives them away from its lower side. If clearly explained, the method of experiment developed and now under study by Dr. Lodge and Mr. Clark, and that of Mr. Aitken on the condensation of water about nuclei, will probably be found productive of results of the very highest importance.

Questions of climate, rainfall, healthfulness of districts, fogs, mists, humidity, \&c., can probably be better studied than in any other way by some form of apparatus based upon results obtained by these experiments, if the theory of flotation above set forth is connected with them, as I trust it may be.

EDW. W. SerRell, Jun.

Chabeuil, Drôme, France, April 27

Mr. Serrell is no doubt perfectly correct in his view that the average spccific gravity of a warmed and vapour-filled cloud may be often less than that of air. The ascent of the so-called "steam" from a kettle proves this, and he will find the view clearly stated in Maxwell's "Heat," p. 280. I did not enter into details in the Dublin lecture, but I was fully convinced of the truth of this statement.

His supposition that the dusty air near a hot body gets warmed not by gaseous conduction from the hot body but by interception of its radiation by the suspended particles, is not an unnatural one, but it is practically untrue. It is disproved by the fact that the concentrated radiation from the electric light is much less effective in warming dusty (or any other) air, than is the neighbourhood of a warm solid only a few degrees above the atmospheric temperature.

Mr. Serrell's criticism, that we do not clearly explain the down-streaming dark plane from a cool body observed by Lord Rayleigh, is quite legitimate. So far as I entered into the matter at all, I intended to indicate provisionally a distinction between a cool body and a very cold one-the boundary coming somewhere, say, between ten and thirty degrees below the air, or possibly depending upon actual temperature as well as on difference. I am not prepared to assert that the bombardment of particles towards a cool body begins the instant it is colder than the atmosphere. I think it possible that there may be a neutral point below which it begins.

But Mr. Clark is working out this among many other points, and I am not sure that his view at present agrees with my hypothesis. Hc will doubtless make a complete statement when ho publishes an account of the quantitative research he is now engaged in. Till then I prefer to leave the account of cold bodies a little vague.

O. J. LODGE

\section{The Supposed Volcanic Dust Phenomena}

THE reddish circle round the sun, which I suppose must be considered as a kind of very large corona, alluded to by $\mathrm{E}$. Divers of Tokio (NATURE, vol. xxix. p. 283), G. F. Burder (p. 525), and other observers, was invariably visible here, when circumstances favoured, from November 1883 up to April 3 . In the middle of that day, and of the $4^{\text {th }}$, though circumstances seemed favourable for seeing it, no tinge of red was perceptible; but it was visible late in the afternoon of the 4 th. Since then it has become more visible again, and from April $2 \mathbf{I}$ has been very plain, though not so conspicuous as it was originally. It is red in the middle of the day, and brown towards sunset, the bright space between it and the sun being blue or greenish.

The semicircle opposite the sun is now far fainter than it was originally, indeed $I$ do not think I should notice it now without looking for it. It is now plainest when the sun is a little above the horizon, which was not formerly the case, and I have not seen it after sunset lately. This may perhaps be owing to a change in the height of the volcanic dust, or whatever it is.

The amount of sediment in the rain strikes me as being very large. I have at different times in the last few months collected it upon glass and examined it with the microscope : there appear in it a considerable variety of crystals and other transparent objects. Some of the crystals are like those drawn by Mr. Beyerinck (vol. xxix. p. 309). I have usually found a number of irregular transparent pieces, but I cannot say that they have 